\title{
The effects of two planning interventions on the oral health behavior of Iranian adolescents: A cluster randomized controlled trial
}

\author{
Amir H Pakpour ${ }^{1}$, Maryam Gholami ${ }^{2}$, Paul Gellert ${ }^{3}$,Mir Saeed yekaninejad ${ }^{4}$, Stephan U \\ Dombrowski ${ }^{5}$, and Thomas L Webb ${ }^{6}$ \\ ${ }^{1}$ Social Determinants of Health Research Center, Qazvin University of Medical Sciences, Shahid Bahounar BLV, \\ Qazvin 3419759811, Iran e-mail: Pakpour Amir@yahoo.com \\ ${ }^{2}$ Department of Psychology, Freie Universitat Berlin, Germany \\ ${ }^{3}$ Charité - Universitätsmedizin Berlin, Germany \\ ${ }^{4}$ Department of Epidemiology and Biostatistics, School of Public Health, Tehran University of Medical Sciences, \\ Tehran, Iran \\ ${ }^{5}$ School of Natural Sciences, University of Stirling, Cottrell Building, FK9 4LA, Stirling, UK \\ ${ }^{6}$ Department of Psychology, University of Sheffield, UK.
}

In press, Annals of Behavioral Medicine, The final publication is available at Springer via https://doi.org/10.1007/s12160-015-9767-3

\section{Conflict of interest and source of funding statement}

The authors declare that they have no conflict of interests. The study was supported by the Department of Public Health at Qazvin University of Medical Sciences 


\section{Acknowledgments}

The authors thank the schools, teachers, and children involved in the study. This study was funded by the Qazvin University of medical sciences, via a Grant to Amir H Pakpour. The authors declare no potential conflicts of interest with respect to the authorship and/or publication of this article. 


\title{
The effects of two planning interventions on the oral health behavior of Iranian adolescents: A cluster randomized controlled trial
}

\begin{abstract}
Purpose To investigate the effectiveness of a planning intervention (specifying when, where, and how to act) and an implementation intention intervention (specifying the same in the format of an if-then plan) in increasing self-reported brushing in adolescents.

Methods The study adopted a cluster randomized controlled trial design and 1,158 students in 48 schools were randomized to planning, implementation intention, or active control conditions. After baseline assessment, all participants received a leaflet containing information and recommendations on oral health and instructions on correct brushing behavior. After reading the leaflets they were provided with a toothbrush and toothpaste plus a calendar in which to record their brushing. Participants in the planning condition and in the implementation intention condition also received instructions to form specific plans regarding brushing behavior. Selfreported brushing, perceived behavioral control, self-monitoring, intention, frequency of planning, oral health-related quality of life, and dental plaque and periodontal status were measured one and six months later.

Results Both intervention conditions showed a significant improvement in the frequency of selfreported brushing, self-monitoring, frequency of planning, intention, perceived behavioral control, plaque index, periodontal health, and oral health related quality of life compared to the control condition at both follow-ups. Comparing the two intervention conditions revealed that adolescents who received the implementation intention intervention had significantly greater
\end{abstract}


improvement in frequency of self-reported brushing, intention, frequency of planning and periodontal health than those in planning condition.

Conclusions Taken together, the findings suggest that forming implementation intentions as well as planning have the potential to increase dental self-reported brushing rates in adolescents, but that forming implementation intentions has the strongest impact on dental hygiene behavior and is, therefore, recommended.

Keywords: Oral health, adolescents, planning, volitional intervention 


\section{Introduction}

Oral diseases, periodontal disease, and tooth loss are an alarming public health problem. Their impact on individuals in terms of pain, impairment of function and reduced quality of life is substantial [1]. Oral diseases can, however, be prevented or reversed by regular performance of oral hygiene behaviors at home (e.g., brushing with fluoride toothpaste twice a day and dental flossing [2]). However, adherence to oral hygiene behaviors is suboptimal with a significant proportion of people brushing and flossing their teeth less than is recommended or needed to prevent problems $[2,3]$.

Various factors influence the likelihood that health behaviors such as oral hygiene behaviors will be performed. One of the strongest predictors is having favorable intentions to perform the behavior $[4,5]$. However, only approximately half of the individuals who express strong intentions to perform a behavior will translate this into behavioral performance [6], leading researchers to talk about a 'gap' between intentions and action $[7,8]$ that needs to be addressed via, for example, volitional interventions. Fortunately, a large body of evidence suggests that forming plans specifying when, where and how to act can increase the likelihood that intentions are successfully translated into action [9, 10]. Planning can enhance the accessibility of relevant cues, forge a mental link between specific cues and behavioral responses and, as a consequence, decrease the likelihood of forgetting or missing opportunities to act [1114].

One critical element on which planning interventions differ is how explicit they are about forging a link between a critical cue and the desired response. Some planning interventions ask participants to specify what, when, where and how to act, without explicitly linking the anticipated critical cues (e.g., the when and where elements) and the response (i.e., the what and 
how elements). In contrast, interventions that ask participants to form implementation intentions typically require that participants specify their plans in an if-then format that explicitly links the anticipated situation (specified in the if-part of the plan) to a specific response (specified in the then-part of the plan) [15]. By explicitly linking cue and response implementation intentions might have an advantage over more general forms of planning. However, although accumulated evidence points to the effectiveness of implementation intentions and planning in increasing health behaviors $[16,17]$, little research to date has systematically compared the effectiveness of different forms of planning interventions within the same study.

\section{Aims and Hypotheses}

The aim of the present research was to test and compare the effects of a planning intervention (specifying when, where, and how to act) and an implementation intention intervention (specifying the same information in a contingent if-then format) on adolescents' dental brushing behavior in a longitudinal, experimental design with one- and six-months followup. It was hypothesized that:

1. Both planning interventions would have a significant effect on behavioral outcomes (e.g., frequency of self-reported brushing), as well as clinical outcomes (e.g., oral health-related quality of life, dental plaque and periodontal status) and psychological outcomes (e.g., proximal determinants of behavior, such as perceived behavioral control) compared with active control condition.

2. The implementation intention intervention will have a significant additional effect on behavioral, clinical, and psychological outcomes compared with the planning intervention. 


\section{Methods}

\section{Design and study population}

The study was conducted in the Qazvin province (Located $150 \mathrm{~km}$ northwest of Tehran), which contains $2 \%$ of the population of Iran. Qazvin has 73 secondary schools within two different educational districts. A list of schools was obtained from the Organization for Education at Qazvin. Schools were eligible for the study if they were situated in Qazvin province and were not currently engaged in an oral health education and promotion program. Five secondary schools were not eligible for the study for these reasons leaving 68 schools for potential inclusion.

The study employed a cluster randomized controlled trial design. There were three conditions (termed 'general planning', 'implementation intention' and 'active control') with data collected at baseline, one and six months. All procedures were carried out in compliance with the Helsinki Declaration. The study was approved by both the Ethics Committee of Qazvin University of Medical Sciences and the Organization for Education at Qazvin. The trial was registered with the ClinicalTrials.gov database (NCT02066987). All participants and their parents provided informed consent prior to participation, and all information about the participants was kept strictly confidential. The CONSORT checklist is available as Checklist S1.

\section{Sample Size}

The sample size was calculated based on the primary outcome measure (self-reported brushing). It was estimated that 370 adolescents would be needed in each condition to detect a medium-sized effect (Cohen's $d=0.50$ ), with 95\% power and a significance level of 5\%, 
assuming an intracluster correlation coefficient of 0.30 , a mean cluster size equal to 25 , with 16 clusters, a 1/3 allocation ratio, and 5\% lost to follow up.

\section{Recruitment}

To account for potential attrition, we aimed to recruit 1,110 students from the 68 eligible schools. According to a report of the Organization for Education at Qazvin, high school classes contained an average of 25 students. Therefore, 48 schools were selected randomly from 68 eligible schools providing a potential yield of around 1200 students. Twenty-four schools were selected randomly from each of the two districts in the recruitment area including twelve boysonly and twelve girls-only schools from each district. An invitation letter was sent to the principals of eligible schools to participate in the study. All 48 eligible schools agreed to participate in the study and the study aims were subsequently communicated to the adolescents and their parents. Recruitment started in February 2014, and 1 month and 6 month follow ups were completed in April 2014 and October 2014. Figure 1 shows the flow of students through the study [25]. A total of 1,308 students were screened for study eligibility. Of the 1,271 eligible students, a total of 1,158 (91.1\%) students in 48 schools participated in the study.

\section{Random Allocation}

Due to the potential for contamination between conditions if students in the same class were allocated to different conditions, stratified cluster randomization was used to assign a classroom from each of the schools into three conditions. Strata's for randomization were education district and gender. That is, from each district, one class from a boys-only and one class from a girls-only school was randomly assigned into one of the three conditions. A 
computer-generated list of random numbers was used by an independent statistician to randomize classes.

\section{Masking}

The research assistants, statisticians and outcome assessors were all masked to condition.

\section{Intervention}

After baseline assessment, all adolescents received a leaflet containing information on oral health, the importance of brushing, and the recommended number of times that people should brush daily. The correct technique for brushing was presented using photos. The modified Bass technique of toothbrushing was described in order to promote plaque removal from both coronal and gingival margins [18]. All adolescents were provided with a toothbrush and toothpaste after reading the leaflets. Finally, the adolescents were provided with a calendar and asked to keep a record of their dental brushing behavior. The intervention was delivered by a health psychologist with a background in oral health (the first author). Participants in the general planning condition then received following instructions:

It may be helpful for you to plan when and where you will brush your teeth each day over the next month. Please write below when, where, and after what activity you will brush your teeth (e.g., at 8.00 a.m. and 9.00 p.m. in the bathroom after eating breakfast/dinner). Because you should brush your teeth twice a day, please make two plans.

I will brush my teeth at (WHEN) at or in (WHERE) before/after (ACTIVITY).

I will brush my teeth at (WHEN) at or in (WHERE) before/after (ACTIVITY). 
Participants in the implementation intention condition received the same instructions, with the exception that the planning exercise was structured in an if-then format:

If it is ___ (WHEN) at or in ___ (WHERE) beforelafter ___ (ACTIVITY), then I will brush my teeth!

If it is ___ (WHEN) at or in ___ (WHERE) beforelafter ___ (ACTIVITY), then I will brush my teet!

Both planning interventions as well as practicing the correct brushing procedure took around 20 minutes to complete. Adolescents in both conditions wrote down their plans and were allowed to take them home. No further intervention was delivered to adolescents in the active-control condition.

\section{Measures}

The primary outcome measure was the frequency of self-reported brushing. Participants were asked to indicate "How many times in the past month have you brushed your teeth?" Previous studies have validated this self-reported measure against clinical indices including periodontal status and dental plaque $[19,20]$.

Secondary outcomes included perceived behavioral control (PBC), self-monitoring of brushing, intentions to brush, frequency of planning, oral health-related quality of life (OHRQOL), and dental plaque and periodontal status.

PBC was assessed using four items (e.g., "It is up to me whether or not I brush my teeth twice a day in the future"). All items were rated on a 5-point scale ranging from 1 (strongly 
disagree) to 5 (strongly agree). Internal reliability was good $(\alpha=0.83)$ and the measures were combined to create a single index.

Self-monitoring was measured by 3 items rated on 5-point scales that ranged from 1 (strongly disagree) to 5 (strongly agree). The item was introduced by the stem "During the last four weeks, I have consistently monitored..." (a) "when to brush my teeth", (b) "how often to brush my teeth", and (c) "how to brush my teeth". Internal reliability was good $(\alpha=0.82)$ and the measures were combined to create a single index.

Behavioral intention was assessed using four items (e.g., "I intend to brush my teeth twice a day in the future"). The items were rated on 5-point scales that ranged from 1 (strongly disagree) to 5 (strongly agree). Internal reliability was good $(\alpha=0.84)$ and the measures were combined to create a single index.

Frequency of planning was assessed by responses to the stem "I have made a detailed plan regarding..." on five 5-point scale that ranged from 1 (strongly disagree) to 5 (strongly agree): (a) "when to brush my teeth", (b) "where to brush my teeth", (c) "how to brush my teeth", (d) "how often to brush my teeth", and (e) "how much time to spend on brushing my teeth". Internal reliability was good $(\alpha=0.82)$ and the measures were combined to create a single index.

The Pediatric Quality of Life Inventory ${ }^{\mathrm{TM}}\left(\mathrm{PedsQL}^{\mathrm{TM}}\right)$ Oral Health Scale was used to examine OHRQOL. The scale comprises five items (e.g.," I have blood on my toothbrush after brushing my teeth"). All responses are reverse scored and transformed into a 0-100-point scale with higher scores representing better OHRQoL. The psychometric properties of the Iranian scale have been described in depth elsewhere [21]. 


\section{Clinical measurements}

Two trained dentists who were masked to the condition allocation examined the clinical oral indices including Visual Plaque Index (VPI) and Community Periodontal Index (CPI). All clinical examinations were conducted during school hours in the health office of the school. Adolescents were sat on a comfortable chair and a mouth mirror attached to an intra-oral LED light and a World Health Organisation periodontal probe was used to assess their oral health.

The VPI was assessed based on Turesky's Modification of the Quigley-Hein Plaque Index with the disclosing agent. To assess VPI, two surfaces were assessed including lingual and buccal. The amount of plaque on each enamel block for each lingual and buccal surface was rated on a six point Likert-type scale ranging from 0 (no plaque) to 5 (plaque covering two-thirds or more of the crown of the tooth). The average score across the two surfaces [22] served as our outcome measure.

Periodontal status was assessed using the CPI. The mouth was divided into sextants and six index teeth (i.e., 16, 11, 26, 36, 31 and 46) were examined. In order to avoid false sacs associated with tooth eruption in children under the age of 15 , pockets were not recorded. The CPI scores were $(0=$ "healthy gingiva", $1=$ "gingival bleeding", 2 = "calculus," 3 = "pocket from 4 to $5 \mathrm{~mm}$ ", and $4=$ "pocket $\geq 6 \mathrm{~mm} "[23])$.

Interrater-reliability and intrarater-reproducibility were assessed in a separate sample of adolescents ( $n=24 ; 12$ boys and 12 girls) prior to the main study. Two dentists rated the adolescents in terms of VPI and CPI. The assessments replicated 1 hour later. Intraclass correlation coefficients (ICC) of the two indices were 0.98 and 0.94 for VPI and CPI, respectively, indicating acceptable interrater reliability. High agreements were found between the 
two assessments of these indices (ICCs ranged from 0.89 to 0.92 for VPI and CPI, respectively) indicating high intrarater- reliability. The same dentists performed all clinical oral exams in the study. Adolescents received $15 \$$ for each of the following study elements: enrollment, session completion, and completion of follow-up surveys.

All measures were taken at baseline, 1 month and 6 months post-intervention.

\section{Data Analysis}

Baseline characteristics of the 48 schools and students were compared using $\chi^{2}$ tests, Fisher's Exact Tests or ANOVA, as appropriate. In order to accommodate the clustering of participants in schools, a sequence of multilevel models (MLM) was used to determine whether significant variation in individual- and school-level outcomes existed across schools. In order to produce unbiased estimates of the random parameters, a restricted iterative generalized least square (RIGLS) estimation procedure was performed. A three-level model was established to estimate change in outcome variables: This model specified repeated assessments (time) at the first level, adolescents at the second level, and schools at the third level. The effect of potentially confounding variables (e.g., age, sex, fathers' level of education) on response variables was measured using univariate multilevel analyses and those variables that had $p$ value less than 0.20 were entered into the multiple variable model (data are not shown).

Five fixed effects were entered for each model; an intercept term, a slope for age (years), a slope for fathers' level of education (years), and dummy variables for gender and condition. To compare the effect of condition at each time point, three interaction terms (condition by time) were estimated for each time point (1 and 6 months) for each response variable (self-reported frequency of brushing, CPI, and VPI). The Benjamini and Hochberg false discovery rate was 
used to adjust $p$-values for multiple comparisons [24]. Analyses were done by intention to treat assuming that missing values were random. Data were analyzed with MLwiN 2.27 software.

\section{Results}

There were no significant differences between the conditions in terms of sociodemographic characteristics as well as the study measures (Table 1). All participants remained in the study at one month post-intervention (Time 2$)$, but a small number of participants $(\mathrm{n}=49$ participants, $4.23 \%$ ) were absent from school at the 6 month follow-up (Time 3). An analysis of dropout by schools did not reveal any pattern of non-participation according to socio-economic status of the school.

Table 2 provides the descriptive statistics for the study outcomes by condition and assessment period. Tables 3, 4, and 5 show the point estimates and standard errors for the effect of condition and other covariates on outcome variables using multilevel mixed model analysis. Variances of second level (student) and third level residuals (school) are presented in all models. Wald tests showed that the second and third level residuals had significant variation after adjusting for independent variables.

\section{Intervention effects on brushing behavior}

Adolescents in the intervention conditions reported brushing more frequently than adolescents in the control condition at both the 1-month and 6-month follow-ups (Table 4). At the 1-month and 6-month time points, the frequency of brushing among adolescents in the implementation intention condition was also significantly higher than among those in the planning condition. 
Intervention effects on intention and $P B C$

As shown in Table 3, adolescents in the implementation intention condition reported stronger intentions to brush in the future than those in the planning condition at the 1-month and six-month assessments. Adolescents in both intervention conditions reported significantly stronger intentions to brush compared to adolescents in the control condition $(p<0.05)$ at the one-month and six-month assessments. Moreover, adolescents in both planning conditions reported more PBC over brushing compared to adolescents in the control condition $(p<0.05)$, but there was no significant differences between the intervention conditions.

Intervention effects on self-monitoring and frequency of planning

Table 3 shows that adolescents in both intervention conditions showed a significant increase in self-monitoring of brushing and more frequent planning compared to adolescents in the control conditions at both 1-month and 6-month follow-ups. At the 1-month and 6-month time points, adolescents in the implementation intention condition reported using planning strategies more frequently compared than those in planning condition. However, there was no significant difference between intervention conditions in self-monitoring over time.

\section{Intervention effects on clinical measurements}

Table 4 compares outcomes between the conditions at each time point after intervention (1 and 6 months) adjusting for age, sex, and father's level of education. There was a significant difference at both time points in VPI between each of intervention conditions and control condition $(p<0.001)$; these indices also differed significantly between the implementation intention condition and the planning condition $(p<0.001)$. There was a significant difference between each of the intervention conditions and control condition in CPI at 6-months $(p \leq 0.001)$ 
and CPI was also significantly lower among adolescents in the implementation intention compared to adolescents in the planning condition $(p<0.001)$. Significant reductions were detected in plaque index and CPI for both intervention conditions over time. With respect to plaque index, VPI was significantly improved among adolescents in both intervention conditions compared with adolescents in the control condition at both follow-ups. Adolescents in the implementation intention condition were more likely to have better dental plaque control and periodontal health at the end of the study than were adolescents in the general planning condition. The ICC (intraclass correlation) between self-reported frequency of brushing, CPI and VPI were $0.31,0.23$ and 0.31 respectively.

Intervention effects on oral health related quality of life

After adjustment for baseline levels, the intervention conditions had significantly higher PedsQL scores than the control condition, suggesting that the interventions positively influenced OHRQoL. There was no significant difference between the implementation intention and planning conditions in terms of OHRQoL. According to this model, the ICC was 0.05 for OHRQoL.

\section{Discussion}

The present research provided one of the first tests of the effects of planning (specifying when, where, and how to act) and forming implementation intentions (specifying the same in an if-then format) on adolescents' dental brushing behavior using an experimental design with one and six months follow-up.

\section{Effect of planning and implementation intentions on dental behavior}


As hypothesized, the planning and implementation intention interventions had a significant effect on self-reported brushing behavior, clinical, and psychological outcomes as compared to an active control condition. This finding is in line with Schüz et al. [26] who found that a brief planning intervention specifying when, where, and how to floss increased flossing compared to a control condition 2 and 8 weeks later. It is worth noting, however, that despite the considerable room for improvement in baseline self-reported brushing behavior in the present research (the baseline frequency of self-reported brushing was below 13 times per month on average), even the highest rate of self-reported brushing post intervention (16 times per month in the implementation intention condition 6 months post intervention) was far from the recommended rate of brushing (approximately 60 times per month) [27]. This observation demonstrates that, although planning and implementation intentions are effective in increasing the target behavior, additional intervention components might be needed in order to achieve the recommended level of behavioral performance.

\section{Effect of planning compared with implementation intentions on dental behavior}

Confirming Hypothesis 2, the implementation intention intervention was significantly more effective in promoting brushing behavior and clinical outcomes. This finding supports the idea (and accumulating evidence) that making plans for action in a contingent if-then format is particularly effective [15]. The difference between forming implementation intentions and more general forms of planning is that good opportunities to act (specified in the if-part of the plan) are explicitly linked to suitable responses to these opportunities (in the then-part of the plan). The consequence is that good opportunities to act are swiftly and accurately identified and intended responses are initiated relatively automatically - that is quickly, efficiently, and without 
deliberation [9]. In contrast, more general forms of planning may serve only to overcome the problem of poorly elaborated intentions [16], rather than instigating cognitive processes that foster effective goal striving. On the basis of the present findings, we therefore recommend that researchers and practitioners interested in promoting oral health in adolescents (and other outcomes) prompt motivated participants to form if-then plans to support their intentions. Studies have shown implementation intentions to be feasible and effective "one minute interventions" which can be delivered without face-to-face contact [28]. The formation of implementation intentions might, therefore, be prompted through filling in electronic or paper-based planning exercises as part of daily clinical routines.

\section{Limitations}

The present research has some limitations. First, our primary outcomes were self-reported measures of oral health behavior, which may be susceptible to bias due to memory or social desirability. However, the clinical measures used in this study showed similar effects as the selfreport measures and previous evidence has attested to the validity of self-report measures of brushing $[19,20]$. Second, the extent to which the obtained effects are sustainable beyond 6 months are unclear. Future studies may benefit from examining effects over even longer time periods. The largest changes occurred between baseline and 1 month follow-up and so future studies should consider maintenance of the effects and how if-then planning can be used to promote habitual behavior and to deal with lapses [29].

\section{Conclusions}

Planning interventions are an economic and effective way to change oral self-care behavior. The findings of this study suggest that specifying when, where, and how to perform the 
goal-directed behavior and, in particular, forming implementation intentions (specifying the above in a contingent if-then format) has the potential to increase dental brushing rates among adolescents. 


\section{References}

1. Petersen PE: World Health Organization global policy for improvement of oral health-World Health Assembly 2007. Int Dent J 2008, 58(3):115-121.

2. Ramsay P: The board of registration in dentistry. How to keep your dental practice out of trouble. J Mass Dent Soc 2000, 49(2):24-26.

3. Schüz B, Sniehotta FF, Wiedemann A, Seemann R: Adherence to a daily flossing regimen in university students: effects of planning when, where, how and what to do in the face of barriers. Journal of clinical periodontology 2006, 33(9):612-619.

4. Ajzen I: The theory of planned behavior. Organ Behav Hum Decis Process 1991, 50:179-211.

5. Triandis HC: Interpersonal behavior. Monterey, CA: Brooks/Cole; 1977.

6. Sheeran P, Orbell S: Do intentions predict condom use? Meta-analysis and examination of six moderator variables. Br J Soc Psychol 1998, 37(2):231-250.

7. Sheeran P: Intention-Behavior Relations: A Conceptual and Empirical Review. Eur Rev Soc Psychol 2002, 12:1-36.

8. Webb TL, Sheeran P: Does changing behavioral intentions engender behavior change? A meta-analysis of the experimental evidence. Psychol Bull 2006, 132:249-268.

9. Gollwitzer PM, Sheeran P: Implementation intentions and goal achievement: A metaanalysis of effects and processes. Adv Exp Soc Psychol 2006, 38:69-119.

10. Sniehotta FF, Araújo Soares V, Dombrowski SU: Randomized controlled trial of a oneminute intervention changing oral self-care behavior. $J$ Dent Res 2007, 86(7):641-645.

11. Webb TL, Sheeran P: How do implementation intentions promote goal attainment? A test of component processes. J Exp Soc Psychol 2007, 43:295-302.

12. Webb TL, Sheeran P: Mechanisms of implementation intention effects: the role of goal intentions, self-efficacy, and accessibility of plan components. Br J Soc Psychol 2008, 47(Pt 3):373-395.

13. Webb TL, Sheeran P: Identifying good opportunities to act: Implementation intentions and cue discrimination. Eur J Soc Psychol 2004, 34:407-419.

14. Gollwitzer PM: Implementation intentions: Strong effects of simple plans. Am Psychol 1999, 54(7):493-503.

15. Oettingen G, Hönig G, Gollwitzer PM: Effective self-regulation of goal attainment. Int $J$ Educ Res 2000, 33:28.

16. Sheeran P, Milne S, Webb TL, Gollwitzer PM: Implementation intentions and health behaviour: Bibliothek der Universität Konstanz; 2005.

17. Hagger MS, Luszczynska A: Implementation Intention and Action Planning Interventions in Health Contexts: State of the Research and Proposals for the Way Forward. Appl Psychol Health Well Being 2014, 6(1):1-47.

18. Poyato-Ferrera M, Segura-Egea JJ, Bullon-Fernandez P: Comparison of modified Bass technique with normal toothbrushing practices for efficacy in supragingival plaque removal. International journal of dental hygiene 2003, 1(2):110-114.

19. Pakpour AH, Sniehotta FF: Perceived behavioural control and coping planning predict dental brushing behaviour among Iranian adolescents. Journal of clinical periodontology 2012, 39(2):132-137. 
20. Pakpour AH, Hidarnia A, Hajizadeh E, Plotnikoff RC: Action and coping planning with regard to dental brushing among Iranian adolescents. Psychology, health \& medicine 2012, 17(2):176-187.

21. Pakpour AH, Yekaninejad MS, Zarei F, Hashemi F, Steele MM, Varni JW: The PedsQL Oral Health Scale in Iranian children: reliability and validity. International journal of paediatric dentistry / the British Paedodontic Society [and] the International Association of Dentistry for Children 2011, 21(5):342-352.

22. Turesky S, Gilmore ND, Glickman I: Reduced plaque formation by the chloromethyl analogue of victamine C. Journal of periodontology 1970, 41(1):41-43.

23. Ainamo J, Barmes D, Beagrie G, Cutress T, Martin J, Sardo-Infirri J: Development of the World Health Organization (WHO) community periodontal index of treatment needs (CPITN). International dental journal 1982, 32(3):281-291.

24. Benjamini Y, Hochberg Y: Controlling the false discovery rate: A practical and powerful approach to multiple testing. J R Stat Soc Ser B Stat Methodol 1995, 57:289-300.

25. Campbell MK, Piaggio G, Elbourne DR, Altman DG, Group C: Consort 2010 statement: extension to cluster randomised trials. Bmj 2012, 345:e5661.

26. Schüz B, Wiedemann AU, Mallach N, Scholz U: Effects of a short behavioural intervention for dental flossing: randomized-controlled trial on planning when, where and how. Journal of clinical periodontology 2009, 36(6):498-505.

27. Pakpour AH, Hidarnia A, Hajizadeh E, Kumar S, Harrison AP: The status of dental caries and related factors in a sample of Iranian adolescents. Medicina oral, patologia oral y cirugia bucal 2011, 16(6):e822-827.

28. Sniehotta FF, Araujo Soares V, Dombrowski SU: Randomized controlled trial of a oneminute intervention changing oral self-care behavior. J Dent Res 2007, 86(7):641-645.

29. Rothman AJ: Toward a theory-based analysis of behavioral maintenance. Health Psychol 2000, 19(1 Suppl):64-69. 


\section{Tables}

Table 1: Demographic characteristics by condition

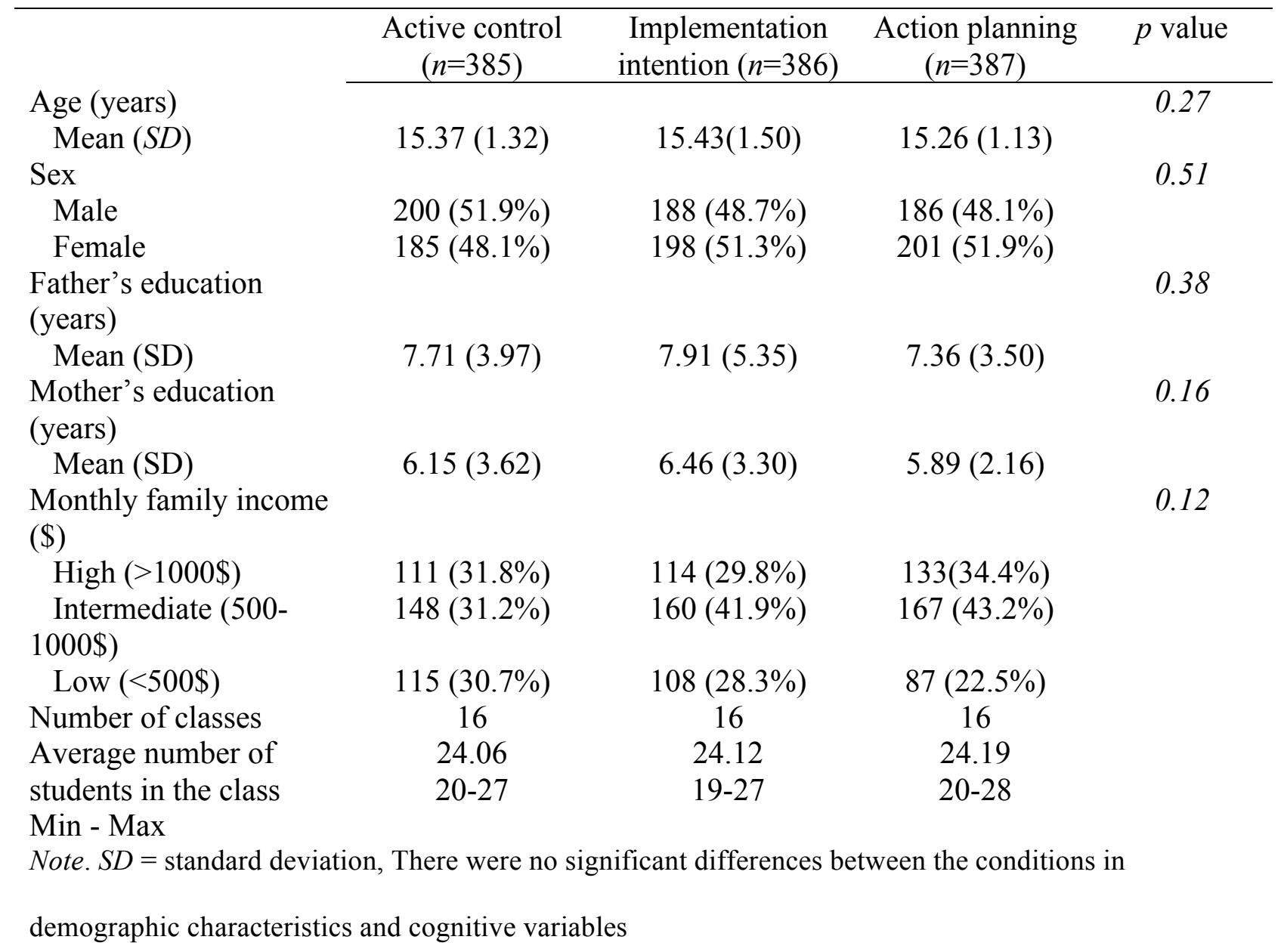


Table 2: Descriptive statistics for all outcome measures by condition and time

\begin{tabular}{lrccc}
\hline Variable & Condition & Baseline & Month 1 & Month 6 \\
Perceived & Active control & $2.63(0.92)$ & $2.68(0.95)$ & $2.63(0.97)$ \\
behavioral control & Imp. intention & $2.59(0.92)$ & $2.86(1.05)$ & $2.82(1.11)$ \\
& Planning & $2.60(0.86)$ & $2.86(1.01)$ & $2.81(1.03)$ \\
Intention & Active control & $2.81(0.68)$ & $2.85(0.70)$ & $2.83(0.71)$ \\
& Imp. intention & $2.82(0.76)$ & $3.42(1.11)$ & $3.40(1.12)$ \\
Self-monitoring & Planning & $2.85(0.80)$ & $3.21(1.05)$ & $3.17(1.05)$ \\
& Active control & $2.00(0.54)$ & $2.29(0.84)$ & $2.27(0.89)$ \\
Planning & Imp. intention & $2.03(0.54)$ & $2.48(1.02)$ & $2.42(1.00)$ \\
& Planning & $2.06(0.63)$ & $2.43(0.86)$ & $2.40(0.86)$ \\
Frequency of & Active control & $1.93(0.57)$ & $1.90(0.57)$ & $1.86(0.57)$ \\
brushing & Imp. intention & $1.90(0.54)$ & $2.56(0.93)$ & $2.55(0.93)$ \\
OHRQoL & Planning & $1.97(0.60)$ & $2.38(0.90)$ & $2.36(0.91)$ \\
& Active control & $12.96(5.46)$ & $13.24(6.41)$ & $13.17(6.47)$ \\
& Imp. intention & $12.90(4.48)$ & $16.07(7.87)$ & $16.00(8.91)$ \\
Community & Planning & $12.91(7.68)$ & $14.86(7.53)$ & $14.75(7.47)$ \\
Periodontal Index & Active control & $79.46(18.20)$ & - & $80.21(18.21)$ \\
(CPI) & Imp. intention & $77.39(22.86)$ & - & $87.30(16.67)$ \\
Visual plaque index & Planning & $79.50(19.20)$ & & $88.10(15.80)$ \\
(VPI) & Active control & $1.75(0.41)$ & $1.72(0.44)$ & $1.71(0.47)$ \\
& Amp. intention & $1.74(0.66)$ & $1.68(0.73)$ & $1.44(0.91)$ \\
\hline Note. Standare control & $2.86(0.85)$ & $2.79(0.90)$ & $2.84(1.00)$ \\
& Planning & $1.75(0.54)$ & $1.66(0.65)$ & $2.18(0.96)$ \\
& Planning & $2.73(0.95)$ & $2.483(0.96)$ & $2.51(1.00)$ \\
\hline
\end{tabular}

Note. Standard deviations are in parentheses. OHRQoL $=$ Oral health-related quality of life. 
Table 3: Three-level multiple linear regression models predicting intention, perceived behavioral control, self-monitoring and the frequency of planning

\begin{tabular}{|c|c|c|c|c|c|c|c|c|c|}
\hline \multirow[t]{2}{*}{ Variable } & \multirow[t]{2}{*}{ Month } & \multicolumn{2}{|c|}{ INT } & \multicolumn{2}{|c|}{ PBC } & \multicolumn{2}{|c|}{ SM } & \multicolumn{2}{|c|}{ PLN } \\
\hline & & $B(S E)$ & $\begin{array}{c}p- \\
\text { value }\end{array}$ & $B(S E)$ & $\begin{array}{c}p- \\
\text { value }\end{array}$ & $B(S E)$ & $\begin{array}{c}p- \\
\text { value }\end{array}$ & $B(S E)$ & $\begin{array}{c}p- \\
\text { value }\end{array}$ \\
\hline IMP & & $\begin{array}{c}0.01 \\
(0.10)\end{array}$ & 0.96 & $\begin{array}{c}0.07 \\
(0.11)\end{array}$ & 0.52 & $\begin{array}{c}0.03 \\
(0.12)\end{array}$ & 0.80 & $\begin{array}{c}0.03 \\
(0.10)\end{array}$ & 0.74 \\
\hline PL & & $\begin{array}{c}0.03 \\
(0.11)\end{array}$ & 0.80 & $\begin{array}{c}0.04 \\
(0.11)\end{array}$ & 0.70 & $\begin{array}{c}0.06 \\
(0.12)\end{array}$ & 0.64 & $\begin{array}{c}0.02 \\
(0.11)\end{array}$ & 0.83 \\
\hline Month & 1 & $\begin{array}{c}0.05 \\
(0.04)\end{array}$ & 0.17 & $\begin{array}{c}0.04 \\
(0.03)\end{array}$ & 0.17 & $\begin{array}{c}0.29 \\
(0.04)\end{array}$ & $<0.001$ & $\begin{array}{c}0.01 \\
(0.04)\end{array}$ & 0.31 \\
\hline $\begin{array}{l}\text { IMP vs } \\
\text { Control }\end{array}$ & 1 & $\begin{array}{c}0.55 \\
(0.05)\end{array}$ & $<0.001$ & $\begin{array}{c}0.23 \\
(0.04)\end{array}$ & $<0.001$ & $\begin{array}{c}0.16 \\
(0.05)\end{array}$ & 0.002 & $\begin{array}{c}0.69 \\
(0.05)\end{array}$ & $<0.001$ \\
\hline $\begin{array}{l}\text { PL vs } \\
\text { Control }\end{array}$ & 1 & $\begin{array}{c}0.32 \\
(0.05)\end{array}$ & $<0.001$ & $\begin{array}{c}0.22 \\
(0.05)\end{array}$ & $<0.001$ & $\begin{array}{c}0.08 \\
(0.04)\end{array}$ & 0.08 & $\begin{array}{c}0.46 \\
(0.06)\end{array}$ & $<0.001$ \\
\hline IMP vs PL & 1 & $\begin{array}{c}0.24 \\
(0.05)\end{array}$ & $<0.001$ & $\begin{array}{c}0.01 \\
(0.04)\end{array}$ & 0.86 & $\begin{array}{c}0.08 \\
(0.05)\end{array}$ & 0.12 & $\begin{array}{c}0.25 \\
(0.05)\end{array}$ & $<0.001$ \\
\hline Month & 6 & $\begin{array}{c}0.02 \\
(0.03)\end{array}$ & 0.39 & $\begin{array}{c}0.00 \\
(0.04)\end{array}$ & 0.96 & $\begin{array}{c}0.23 \\
(0.04)\end{array}$ & $<0.001$ & $\begin{array}{c}0.07 \\
(0.04)\end{array}$ & 0.07 \\
\hline $\begin{array}{l}\text { IMP vs } \\
\text { Control }\end{array}$ & 6 & $\begin{array}{c}0.56 \\
(0.06)\end{array}$ & $<0.001$ & $\begin{array}{c}0.24 \\
(0.04)\end{array}$ & $<0.001$ & $\begin{array}{c}0.13 \\
(0.06)\end{array}$ & 0.01 & $\begin{array}{c}0.72 \\
(0.06)\end{array}$ & $<0.001$ \\
\hline $\begin{array}{l}\text { PL vs } \\
\text { Control }\end{array}$ & 6 & $\begin{array}{c}0.30 \\
(0.05)\end{array}$ & $<0.001$ & $\begin{array}{c}0.22 \\
(0.05)\end{array}$ & $<0.001$ & $\begin{array}{c}0.11 \\
(0.05)\end{array}$ & 0.04 & $\begin{array}{c}0.46 \\
(0.06)\end{array}$ & $<0.001$ \\
\hline IMP vs PL & 6 & $\begin{array}{c}0.26 \\
(0.05)\end{array}$ & $<0.001$ & $\begin{array}{c}0.01 \\
(0.05)\end{array}$ & 0.82 & $\begin{array}{c}0.05 \\
(0.05)\end{array}$ & 0.31 & $\begin{array}{c}0.25 \\
(0.07)\end{array}$ & $<0.001$ \\
\hline Age & & $\begin{array}{c}0.02 \\
(0.02)\end{array}$ & 0.37 & $\begin{array}{c}0.00 \\
(0.02)\end{array}$ & 0.92 & $\begin{array}{c}0.02 \\
(0.02)\end{array}$ & 0.11 & $\begin{array}{l}-0.01 \\
(0.01)\end{array}$ & 0.005 \\
\hline $\begin{array}{l}\text { Female (vs. } \\
\text { Male) }\end{array}$ & & $\begin{array}{l}0.049 \\
(0.08)\end{array}$ & 0.55 & $\begin{array}{c}0.48 \\
(0.09)\end{array}$ & 0.57 & $\begin{array}{l}-0.01 \\
(0.00)\end{array}$ & 0.05 & $\begin{array}{c}0.32 \\
(0.08)\end{array}$ & $<0.001$ \\
\hline $\begin{array}{l}\text { Father } \\
\text { Education }\end{array}$ & & $\begin{array}{c}0.00 \\
(0.01)\end{array}$ & 0.87 & $\begin{array}{c}0.01 \\
(0.01)\end{array}$ & 0.02 & $\begin{array}{c}0.40 \\
(0.09)\end{array}$ & $<0.001$ & $\begin{array}{c}0.00 \\
(0.01)\end{array}$ & 0.66 \\
\hline Intercept & & $\begin{array}{c}2.34 \\
(0.29)\end{array}$ & $<0.001$ & $\begin{array}{c}2.32 \\
(0.34)\end{array}$ & $<0.001$ & $\begin{array}{c}2.24 \\
(0.25)\end{array}$ & $<0.001$ & $\begin{array}{c}1.96 \\
(0.28)\end{array}$ & $<0.001$ \\
\hline$\hat{\sigma}_{\text {st }}^{2}$ (student) & & $\begin{array}{c}0.45 \\
(0.02)\end{array}$ & $<0.001$ & $\begin{array}{c}0.66 \\
(0.03)\end{array}$ & $<0.001$ & $\begin{array}{c}0.25 \\
(0.02)\end{array}$ & $<0.001$ & $\begin{array}{c}0.39 \\
(0.02)\end{array}$ & $<0.001$ \\
\hline$\hat{\sigma}_{s c}^{2}$ (school) & & $\begin{array}{c}0.06 \\
(0.02)\end{array}$ & $<0.001$ & $\begin{array}{c}0.06 \\
(0.02)\end{array}$ & 0.002 & $\begin{array}{c}0.09 \\
(0.02)\end{array}$ & $<0.001$ & $\begin{array}{c}0.06 \\
(0.02)\end{array}$ & $<0.001$ \\
\hline
\end{tabular}

Note. $\mathrm{IMP}=$ Implementation intention condition. $\mathrm{PL}=$ Planning condition. $\mathrm{INT}=$ intention. $\mathrm{PBC}=$ Perceived behavioral control. $\mathrm{SM}=$ Self-monitoring. $\mathrm{PLN}=$ Frequency of planning. 
Table 4. Three-level multiple linear regression models predicting brushing behavior, periodontal status and plaque index

\begin{tabular}{|c|c|c|c|c|c|c|c|}
\hline \multirow[t]{2}{*}{ Variable } & \multirow[t]{2}{*}{ Month } & \multicolumn{2}{|c|}{ BEH } & \multicolumn{2}{|c|}{ CPI } & \multicolumn{2}{|c|}{ VPI } \\
\hline & & B (SE) & P-value & $\mathrm{B}(\mathrm{SE})$ & P-value & $\mathrm{B}(\mathrm{SE})$ & $P$-value \\
\hline IMP & & $0.04(0.73)$ & 0.95 & $0.01(0.10)$ & 0.95 & $-0.10(0.25)$ & 0.69 \\
\hline PL & & $0.06(0.75)$ & 0.93 & $0.017(0.10)$ & 0.87 & $-0.18(0.26)$ & 0.49 \\
\hline Month & 1 & $1.94(0.15)$ & $<0.001$ & $-0.02(0.02)$ & 0.30 & $-0.07(0.04)$ & 0.08 \\
\hline IMP vs Control & 1 & $2.88(0.22)$ & $<0.001$ & $-0.04(0.03)$ & 0.17 & $-0.54(0.06)$ & $<0.001$ \\
\hline PL vs Control & 1 & $1.66(0.21)$ & $<0.001$ & $-0.07(0.03)$ & 0.01 & $-0.18(0.06)$ & 0.003 \\
\hline IMP vs PL & 1 & $1.22(0.21)$ & $<0.001$ & $0.03(0.03)$ & 0.38 & $-0.37(0.05)$ & $<0.001$ \\
\hline Month & 6 & $1.84(0.15)$ & $<0.001$ & $-0.04(0.03)$ & 0.12 & $-0.08(0.04)$ & 0.06 \\
\hline IMP vs Control & 6 & $2.92(0.22)$ & $<0.001$ & $-0.27(0.03)$ & $<0.001$ & $-0.53(0.05)$ & $<0.001$ \\
\hline PL vs Control & 6 & $1.62(0.22)$ & $<0.001$ & $-0.12(0.04)$ & 0.001 & $-0.15(0.06)$ & 0.02 \\
\hline IMP vs PL & 6 & $1.30(0.21)$ & $<0.001$ & $-0.14(0.03)$ & $<0.001$ & $-0.38(0.06)$ & $<0.001$ \\
\hline Age & & $-0.03(0.13)$ & 0.84 & $-0.02(0.01)$ & 0.22 & $0.02(0.03)$ & 0.39 \\
\hline Female (vs. Male) & & $2.50(0.59)$ & $<0.001$ & $-0.18(0.08)$ & 0.03 & $-0.16(0.21)$ & 0.45 \\
\hline Father Education & & $0.04(0.04)$ & 0.28 & $0.00(0.00)$ & 0.62 & $-0.00(0.01)$ & 0.71 \\
\hline Intercept & & $7.50(2.12)$ & $<0.001$ & $2.09(0.22)$ & $<0.001$ & $3.31(0.47)$ & $<0.001$ \\
\hline$\hat{\sigma}_{\text {st }}^{2}$ (student) & & $6.59(1.20)$ & $<0.001$ & $0.23(0.01)$ & $<0.001$ & $0.99(0.05)$ & $<0.001$ \\
\hline$\hat{\sigma}_{s c}^{2}($ school $)$ & & $3.00(0.85)$ & $<0.001$ & $0.07(0.02)$ & $<0.001$ & $0.45(0.10)$ & $<0.001$ \\
\hline
\end{tabular}

Note. IMP $=$ Implementation intention condition. $\mathrm{PL}=$ Planning condition. $\mathrm{BEH}=$ Frequency of brushing behavior. $\mathrm{CPI}=$ Community Periodontal Index. VPI = visual plaque index. 
Table 5. Three-level multiple linear regression models predicting oral health related quality of life

\begin{tabular}{lccc}
\hline & Month & OHRQOL & \\
& & $\mathrm{B}(\mathrm{SE})$ & -value \\
\cline { 2 - 4 } IMP & & $2.26(1.71)$ & 0.19 \\
PL & 6 & $0.09(1.74)$ & 0.96 \\
Month & 6 & $0.76(0.89)$ & 0.40 \\
IMP vs Control & 6 & $9.15(1.26)$ & $<0.001$ \\
PL vs Control & 6 & $7.85(1.26)$ & $<0.001$ \\
IMP vs PL & & $1.31(1.26)$ & 0.30 \\
Age & & $-0.79(0.38)$ & 0.040 \\
Female (vs. Male) & & $2.15(1.31)$ & 0.10 \\
Father Education & & $0.27(0.12)$ & 0.02 \\
Intercept & & $88.45(6.07)$ & $<0.001$ \\
$\hat{\sigma}_{\text {st }}^{2}$ (student) & & $173.43(11.09)$ & $<0.001$ \\
$\hat{\sigma}_{s c}^{2}$ (school) & & $9.85(4.19)$ & 0.02 \\
\hline Note. IMP $=$ Impla
\end{tabular}

Note. IMP $=$ Implementation intention condition. $\mathrm{PL}=$ Planning condition. $\mathrm{OHRQOL}=$ Oral health related quality of life. 


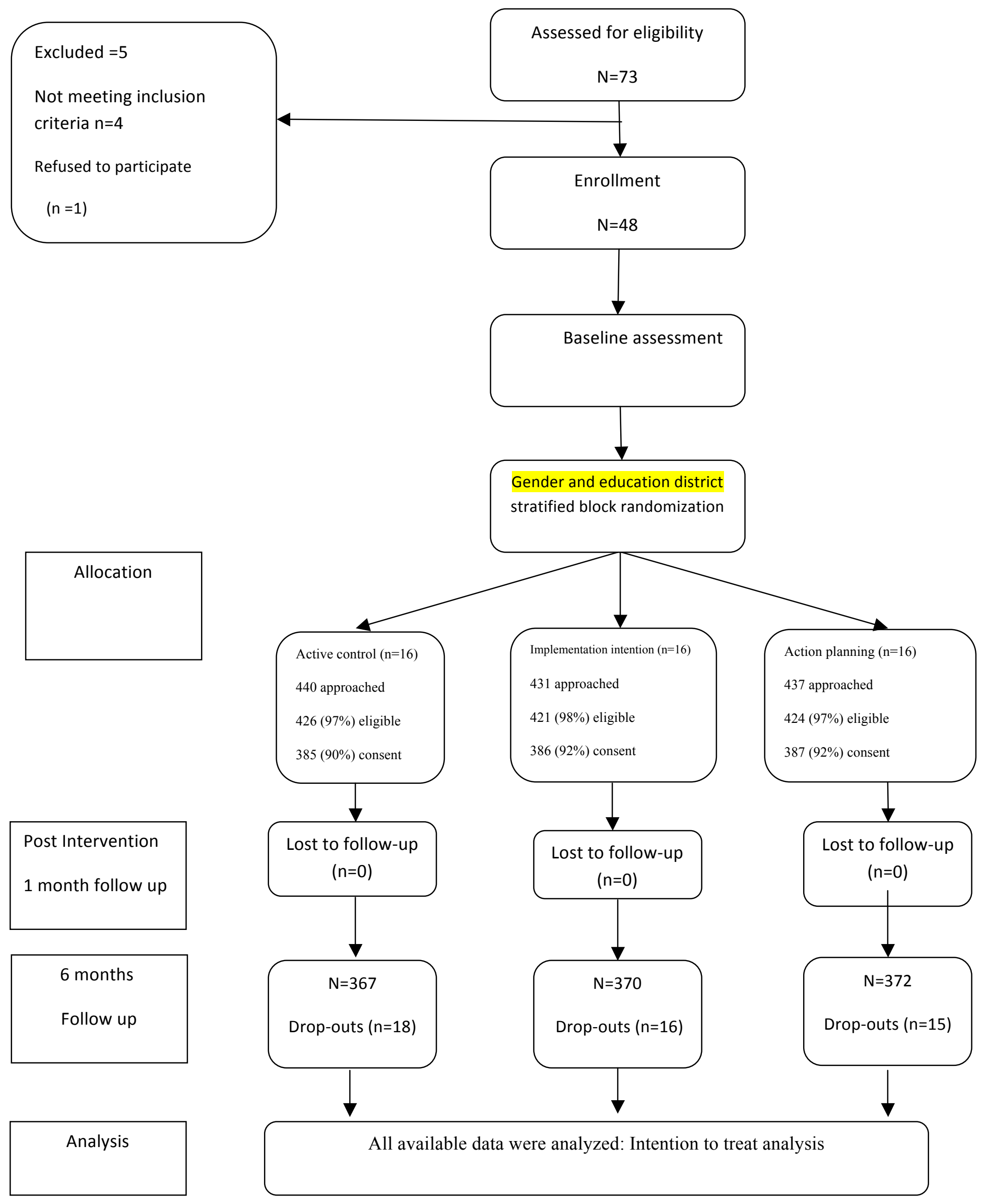

